\title{
Evaluation of Economic Transformation in Coal Resource Type City - A Case Study of Tongchuan City
}

\author{
Lanqin Xing \\ Shanxi Xueqian Normal University, Xi’an, Shanxi, 710100
}

Keywords: coal - based cities, transition degree, Tongchuan City

\begin{abstract}
The paper explores the characteristics of coal-based cities transition degree. Based on the concept of transition degree, this paper constructs the evaluation index system of urban transition degree in Tongchuan City, calculates and standardizes the rate of change based on the relevant raw data from 2006 to 2015 in Tongchuan City, and uses the analytic hierarchy process and entropy method to determine the evaluation index weights, with using the weighted sum to calculate the degree of transition index. Tongchuan transformation system and transformation indicators have different effects on the transformation degree. Tongchuan City, the comprehensive index of economic transformation showed a "W" -shaped volatility characteristics.
\end{abstract}

\section{Introduction}

The transformation of resource-based cities is a worldwide problem and also a realistic need to achieve sustainable development. How to quantitatively analyze and judge the effect of resource-based city transformation and scientifically analyze the connotation of transition has drawn the attention of domestic and foreign scholars. The concept of "transition degree" has been proposed [1-2]. Domestic scholars have also conducted a study of the degree of transition [3-5] However, the evaluation of the passive transition degree of declining coal resources cities is still blank. However, there is no research on the connotation of transformation. This research constructs the evaluation index system and the transition degree evaluation model of transition degree of Tongchuan City, and analyzes the data of Tongchuan City from 2006 to 2015 to explore the relationship between Tongchuan the five characteristics of urban transformation and development provide reference for the connotation measurement research on the transformation and development of other regions.

\section{Tongchuan Overview}

Since 2009, Tongchuan City has been listed as a pilot city for resource-based sustainable development in China, it has entered a period of passive transformation and development with the help of policy support from all levels of government. The National Sustainable Development Plan for Resource-based Cities (2013-2020) listed Tongchuan City as one of 24 resource-degraded prefecture-level administrative regions nationwide. Tongchuan City, the transformation and development of nearly 10 years made some.

However, there are also some problems in the transition process. Therefore, the research on the transition degree in Tongchuan City is the key to analyze the procedural characteristics of Tongchuan transition.

\section{Tongchuan City Economic Transition Evaluation Index System}

To measure the characteristics of regional transformation and development system, we must analyze the degree of transition. Regional transition includes speed, breadth, depth, direction, and easiness [3]. The transition speed measures the speed of transition, taking the economic speed as the performance characteristic. The transformation breadth is a measure of the industrial sector and the adjustment of the industrial structure involved in the transition, which is manifested by the 
proportion of the three industries. The transformation depth is based on the deepening analysis of the transformation breadth, the changes in the proportion of the three industries employed and the pull-in rate of tertiary industries to GDP are the two indicators. The transition direction is the path and mode of the transformation, which is usually analyzed by the leading industries in transition. The easiness of the transition is the analysis of the elements of the transition support, which mainly analyzes the conditions of the transition. Usually, it takes the indicators of human resources and scientific and technological resources required for the transition.

According to the actual Tongchuan City, following the scientific, measurability and accessibility of indicators, the index system of economic transformation evaluation system of Tongchuan is constructed [9-11].

The transformation speed system needs relevant speed indicators to reflect. Taking GDP, per capita GDP and local fiscal revenue as source data, annual change rate of GDP, annual change rate of per capita GDP and annual change rate of local fiscal revenue of Tongchuan City are taken as measurement indexes.

The breadth of the system is measured based on the output value of the industrial structure, that is, the annual change rate of the added value of the three major industries in the GDP. Namely, the annual change rate of the added value of the primary industry, the annual change rate of the added value of the secondary industry and the annual change rate of the added value of the tertiary industry.

The depth of the transformation is guaranteed by changes in the employment structure driven by industrial restructuring and the pulling rate of industrial GDP. That is, the annual rate of change in the proportion of three-time employment in the exhibition industry and the rate of annual change in the rate of three-time industrial pull-in in GDP.

The system of transformational orientation is determined by the basis of the transformation and the objective of the transformation. 2009 Tongchuan build well-known national leisure and health city and livable green ecological livable city, the development of tourism and modern agriculture as the goal. Therefore, the indices of Tongchuan transitional system include annual rate of urbanization, annual change rate of urban GDP to GDP, ratio of industrial output of agriculture, forestry, husbandry and fishery to total output value of agriculture, forestry, animal husbandry and fishery, annual rate of change of per capita net income of farmers, The annual rate of change of domestic and international tourism receipts in GDP, the annual rate of change in the gross value of industrial output above designated size, and the annual rate of change in the overall labor productivity index are all seven indicators.

Easy transition system is determined by the transition resources. The transition system of Tongchuan City includes the annual rate of change of economic external dependence (the degree of foreign dependency is the ratio of total import and export as a percentage of GDP), the annual rate of change of investment in education funds in GDP, the annual energy consumption per unit of GDP Rate of change indicator.

The basic data of the selection index of the article are mainly from Tongchuan Statistical Yearbook, Tongchuan Environmental Gazette, Tongchuan National Economic and Social Development Bulletin, Statistical Region of Shaanxi Province and Statistics of Shaanxi Province from 2006 to 2016 Yearbook "and so on. Tongchuan City transition evaluation system indicators reflect the dynamic change trend of the rate of change indicators, the actual data in this article is based on the original data for the ring rate of change in the data in 2006 as the base 2005 data, the other were The calculation of the above year as the base period is shown in Table 1-1.

\section{Evaluation Results}

According to the actual situation of economic transition in Tongchuan City, the weight analysis of AHP and entropy method shows that the weight of economic system of Tongchuan is 0.3667 , which is the leading system of Tongchuan City's transition. Followed by a system of weighted value of 0.2539 degree system and the weight value of 0.2375 transformation depth system, we can see that the economic transformation and transformation of Tongchuan model is the key to the 
adjustment of industrial structure is the fundamental. The Tongchuan transition speed system and the transition breadth system with weight values of 0.0704 and 0.0714 respectively have little effect on their transition. In short, for the transformation of coal-based Tongchuan, the transformation of human resources and upgrading of science and technology resources are the core of the transformation. Vigorously develop the tourism industry, upgrade the level of urbanization, and promote the development of agricultural service industry is the direction of transformation. The total economic output and the increase of the industrial sector can not be the basis for the internal assessment of the quality of industrial restructuring in Tongchuan.

According to Table 1, Xi'an Municipality has a year-on-year rate of change (0.2402) in investment in fixed assets, an annual rate of change in energy consumption per unit of GDP (0.0917), and an annual rate of change in GDP (0.0907), annual change rate of urbanization level (0.0901), annual change rate of urban GDP in GDP (0.0609), annual change rate of employment in tertiary industry (0.0594), annual change rate of proportion of added value of tertiary industry (0.0438), The annual rate of change of domestic and international tourism receipts as a percentage of GDP (0.0404) shows that the increase of investment in education has led to the improvement of the quantity and quality of human resources, the development of energy saving in low-carbon industries, the expansion of employment in the tertiary sector Industrial output value, increase the level of urbanization and the proportion of urban GDP is the key move and support for the transformation and development of Tongchuan City to the intension-type transformation.

The transition speed system index stabilized before 2011 and then declined year by year. Tongchuan had a small economic aggregate before 2006, with rapid economic growth at a rapid growth rate supported by various levels of policies. In 2009, the economic growth rate of pilot cities designated as resource-based transitions accelerated but policy-oriented economic restructuring support slowed down after 2011 In addition, with the improvement of economic output, the pace of transformation has been slow to grow.

System widening volatility before 2013 upward trend, the index dropped sharply in 2014 and rebounded in 2015. The extent of the transformation is mainly determined by the added value of the industrial structure. Since Tongchuan's industrial structure has been continuously adjusted since 2006, the proportion of added value has been changing steadily. However, changes in statistical policies in 2014 combined with the newly-added increase in industrial output have led to a decline in the index. The exponential growth in 2015 indicates that the system of Tongchuan transition has been improved and is in line with the trend characteristics of the transformation and development.

Table 1. The transition degree index and comprehensive index of Tongchuan City.

\begin{tabular}{l|c|r|r|r|r|r|r|r|r|r}
\hline Year & 2006 & 2007 & 2008 & 2009 & 2010 & 2011 & 2012 & 2013 & 2014 & 2015 \\
\hline Speed (S) & 0.0612 & 0.0672 & 0.0595 & 0.0633 & 0.0653 & 0.0677 & 0.0439 & 0.0472 & 0.0089 & 0.0073 \\
\hline Breadth (B) & 0.0472 & 0.0514 & 0.0573 & 0.0602 & 0.0433 & 0.0626 & 0.0436 & 0.0638 & 0.0116 & 0.0437 \\
\hline depth (D) & 0.0544 & 0.0958 & 0.0431 & 0.1262 & 0.0711 & 0.0837 & 0.0515 & 0.0843 & 0.0372 & 0.1610 \\
\hline Degree (D) & 0.0791 & 0.0950 & 0.2001 & 0.1792 & 0.1667 & 0.1263 & 0.1611 & 0.0829 & 0.0779 & 0.0946 \\
\hline $\begin{array}{l}\text { Easy Index } \\
\text { (D) }\end{array}$ & 0.2537 & 0.0609 & 0.0439 & 0.0663 & 0.1015 & 0.0356 & 0.0196 & 0.0669 & 0.1039 & 0.0990 \\
\hline $\begin{array}{l}\text { Transition } \\
\text { Index }\end{array}$ & 0.4956 & 0.3703 & 0.4038 & 0.4953 & 0.4480 & 0.3759 & 0.3198 & 0.3450 & 0.2395 & 0.4057 \\
\hline
\end{tabular}

The SWIFT index rose before 2009, declining from 2009 to 2014 and rebounded in 2015. From the transformation depth system index, the transformation depth system focuses on the transformation of the connotation transformation. In light of the change in the system index of transition scale, Tongchuan City, although its industrial structure has been continuously adjusted and upgraded in 2006, the economic pull-in rate of the three major industries is still obviously insufficient.

The index of transition system index first increased and then decreased, reaching its peak in 2008. The system of transformation reflects the mode of transformation. In the early transition, urbanization developed rapidly, urban GDP increased rapidly, and the proportion of added value of 
agriculture, forestry, animal husbandry and fishery services changed greatly. However, the transformation mode is only the direction of the transition can not determine the success or failure of the transition, the success or failure of the transition depends on Tongchuan City, human resources, ecology and other resource conditions and the number of external customers and customer spending potential.

The transition system index has been at a low level since 2006, showing a slight increase from 2012 onwards. The change of Tongchuan transitional easiness index is related to the relatively lower education level and the innovation ability of science and technology in Tongchuan, due to the low degree of extroversion of the characteristic economy of coal resource cities. With the increasing investment in education, the overall level of education, the improvement of science and technology, the continuous transformation of human resources and scientific and technological resources have been continuously optimized, and the index of ease of transition has been continuously increasing.

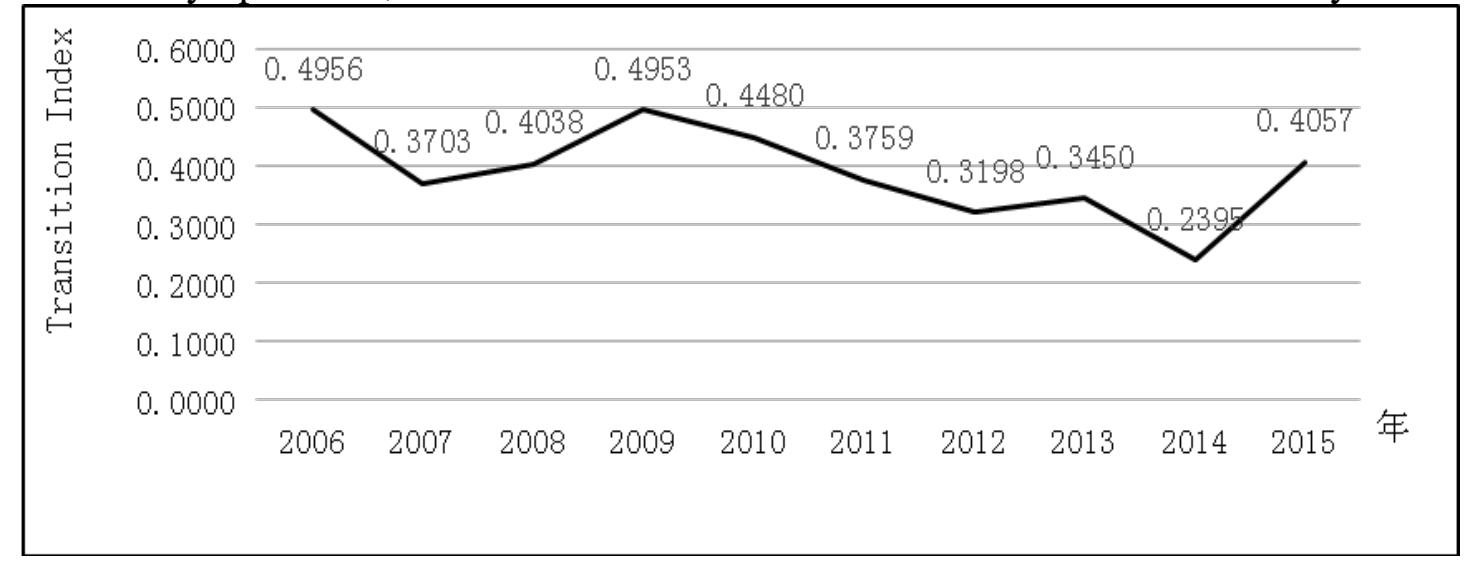

Figure 1. Transition degree of the comprehensive index changes map of Tongchuan City.

Figure 1 shows that the comprehensive index of transition degree of Tongchuan city shows the characteristics of "W" type fluctuation. According to the characteristics of volatility data, it is divided into two stages of transition degree decreasing phase and three high transition degree.

In 2007, 2008 and 2009, Tongchuan's transition index continued to increase. At this stage, Tongchuan City is undergoing a positive transformation and achieved initial success. This phase is a stage of steady transition in which Tongchuan itself is seeking a path of sustainable development and actively undertaking industrial restructuring. The economy However, at this stage, the extent of transformation has been obviously improved. The degree of transformation is not clear, the depth of transformation has not been improved, and the ease of transition has become an obstacle.

Tongchuan City in 2010 a clear goal of transformation, a firm attitude of transformation. However, after the extensive transformation in 2007-2009, it entered a deep transformation, clarified the direction of transformation, and intensified the transformation depth. The transition is low. Although there is policy support at this stage, it is difficult to upgrade the connotation of the transformation and there is not enough potential for transformation.

The composite indices for the transformation in 2006, 2009 and 2015 were $0.4956,0.4953$ and 0.4057 , respectively. These three years are the three years with the highest transition degree between 2006 and 2015 as the year of high transition. The high transition year is based on the largest change in transition during the previous year. In 2005 Tongchuan economy and society in all aspects of the low level, but in 2006 a sudden increase in investment in education, industrial restructuring led to high degree of restructuring index in 2006, Tongchuan City in 2009 was listed as the first year of transition pilot cities, passive policy transformation efforts, Relative to 2008 high transition year. After the precipitation growth in 2010-2014, industrial and industrial restructuring improved, and 2015 reached the year of high transition.

\section{Acknowledgements}

(1) Major Theoretical and Practical Issues in Social Sciences in Shaanxi Province Research 
Project: Research on Functional Division and Coordinated Development Mechanism of Guanzhong Plain Urban Agglomerations (2017Z046).

(2) 2016 China Silk Road Research Institute Scientific Research Projects: Research on the Mechanism and Mode of Cooperation and Linkage of Guanzhong Region and Regional Industries along the Silk Road in the Competition (2016SY05).

\section{References}

[1] Zhang Tingwei, Wu Haojun. Footprint of Transformation: Urban Development and Evolution in Southeast Asia [M]. Nanjing: Southeast University Press, 2008.

[2] Li Xuexin, Tian Guangzeng, Miao Changhong. Economic Transformation of Regional Central Cities: Mechanism and Model [J]. Urban Development Research, 2010, 17 (4): 26-32.

[3] Xu Mei, Liu Chunlao. Connotation and Evaluation of the Transformation of Regional Economy - A Case Study in Western Hunan [J]. Chinese Journal of Natural Resources, 2015, 30(10): 1675-1685.

[4] Zhu Hongrui, Liu Shishun, Qiu Li. Resource-based cities industrial transformation degree of evaluation and case [J]. Journal of Hebei Polytechnic University, 2008, (02): 58-61 + 64.

[5] Wu Jiuxing, Zhao Jie. Anhui Province Rural Transition and Regional Differentiation Characteristics [J]. Hubei Agricultural Sciences, 2017, 56 (13): 2548-2552.

[6] Zhang Fan, Cao Mingming. Study on the Industrial Transformation of Resource-exhausted Cities - A Case Study of Tongchuan City [J]. Journal of Northwest University (Natural Science Edition), 2012, 42 (05): 833-837.

[7] Liu Yansui, Zhang Wenzhong. On the Strategic Choice of Sustainable Development of Mining Cities in West China - - A Case Study of Tongchuan City [J]. Journal of Northwest University (Natural Science Edition), 2001, (01): 84-89.

[8] Liu Rong, Shi Xingmin, Liu Song. Study on Measures and Countermeasures for Sustainable Development of Tongchuan City [J]. Journal of Northwest University (Natural Science Edition), 2011, 41 (05): 901-906.

[9] Che Xiaochai, Zhang Pingyu. The Measurement and Evaluation of Economic Transformation Performance of Resource-based Cities Based on AHP [J]. Journal of Anhui Agricultural Sciences, 2011, 39 (01): 622-624 + 627.

[10] Xu J. Resource-based City Industrial Transformation Evaluation Index System [J]. The Friends of Accounting, 2011, (14): 36-37.

[11] Gu Guofeng. Research on the Construction and Operation Mechanism of Regional Economic Development Power System [J]. GEOGRAPHICAL SCIENCE, 2008, (03): 320-324.

[12] Ni Jiupai, Li Ping, Wei Chaofu, Xie Deti. Evaluation of potential for development and consolidation of regional land based on AHP and entropy weight method [J]. Journal of Agricultural Engineering, 2009, 25 (05): 202-209. 